\title{
Additive Manufacturing- An Enigma: the Future of Oral \& Maxillofacial Surgery
}

\author{
Dr. Ramesh Ram Fry ${ }^{1}$, Dr. Isha Gargya ${ }^{2}$, Dr. Samta Goyal ${ }^{3}$, Dr. Jatinder Pal \\ Singh Chawla ${ }^{4}$, Dr. Pavneet Kaur Pandher ${ }^{4}$, Gurmukh Dhaliwal ${ }^{5}$, Pritpal Singh \\ Ghotra $^{5}$ \\ ${ }^{I}$ Professor, Department Of Oral \& Maxillofacial Surgery, M.M. College Dental Sciences \& Research, Mullana, \\ Ambala, Haryana, India \\ ${ }^{2}$ Senior Lecturer, Department Of Orthodontics \& Dentofacial Orthopedics, Bhojia Dental College \& Hospital, \\ Baddi, Nalagarh, Himachal Pradesh. \\ ${ }^{3}$ Professor, Department Of Oral \& Maxillofacial Surgery, Rayat Bahra Dental College \& Hospital, V.P.O- \\ Sahauran, DISTT. Mohali, Punjab, India. \\ ${ }^{4}$ MDS, Department Of Oral \& Maxillofacial Surgery, M.M. College Dental Sciences \& Research, Mullana, \\ Ambala, Haryana, India \\ ${ }^{5}$ BDS Student, M.M. College Dental Sciences \& Research, Mullana, Ambala, Haryana, India
}

\begin{abstract}
Pertaining to the radical changes that our generation has seen in the past century, the advent of additive manufacturing has initiated a novel approach to use biomaterials. The unprecedented growth in this sector has forced us to realize the application of this technology in dentistry. From its use to print $3 D$ scaffolds for bone tissue engineering to its use in cranial reconstruction, the opportunities are endless. This technology shall bridge the gap by customizing the dentistry we practice till date. And hence we may look forward to a revolutionized $3 D$ printed digital future. This review focuses on the advances in Additive Manufacturing and its growing application in the field of Dentistry with special reference to oral \& maxillofacial surgery
\end{abstract}

\section{Introduction}

The concept of Additive Manufacturing (AM) was first introduced by Chuck Hull in 1986 via a process known as 'stereolithography (SLA)'. ${ }^{[1,2]}$ Additive Manufacturing works on the principle of an inkjet printer. Layer by Layer is generated from a computerized input are laid down in slices to produce a 3 Dimensional object.

The American Society for Testing and Materials (ASTM) defines Additive Manufacturing as:

The process of joining materials to make objects from 3D model data, usually layer upon layer, as opposed to subtractive manufacturing methodologies.

A few of the AM technologies that have found their use in dentistry are:

$>$ 3D printing

$>$ Stereolithography (SLA).

$>$ Fused deposition modelling (FDM).

$>$ Selective Laser Sintering ${ }^{[8]}$

Different AM approaches, for example, 3D Printing (3DP), Solid Freedom Fabrication (SFF), and Rapid Prototyping (RP) use Computer Aided Design (CAD) to print complex shapes for scaffold fabrication ${ }^{[3-5]}$ Some commercially available techniques that print 3D Scaffolds layer-by-layer are 3DP (ExOne, PA),fused deposition modelling (FDM, Stratasys, MN), selective laser sintering(SLS,3D Systems ,CA), Stereolithography (3D systems, CA),3D plotting ( Fraunhofer Institute for Materials Research and Beam Technology, Germany), as well as various forms of direct writing ${ }^{[5]}$

\section{D Printing (3DP)}

In early 1990s at MIT (Cambridge, MA) Sachs et al developed a technology which they named 3DP.

${ }^{[8]}$ This uses a regular inkjet print head and is a powder-based freeform fabrication method. Binders are printed on to lose powders in a powder bed. Early research in this area was focused on rapid tooling using metals and ceramics. ${ }^{[9]}$ Based on the CAD file, the printer sprays the binder across the build layer. The binder, which can be organic or water-based, locally binds the particles and hardens the wetted area, or results in a reaction similar to the hydraulic setting reaction in cements. ${ }^{[10-12]}$ After printing, the printed layer is moved under a strip heater to allow the binder to dry out and prevent spreading between layers. ${ }^{[10]}$ This is followed by 
depowdering which is the removal of loose powder from the printed body.

Application: this technique is most commonly used to print 3D scaffolds for bone tissue engineering. In bone tissue engineering the advantages of this method arise through the control of fine features including interconnected porosity, no contamination issues related to any second material for support structures and the direct printing ability with both metallic and ceramic biomaterials. ${ }^{[12,13]}$

\section{Stereolithography (SLA)}

The first of additive techniques, Hull introduced it to print objects by depositing material rather than removing it. This process involves the immersion of platform in a photopolymer liquid which is then exposed to focused light according to desired design. The polymer solidifies at focal point while the non-exposed polymer remains liquid. Layer by layer the platform is fabricated which moves in a downward direction. The advantages of this method are that complex internal features can be obtained. Growth factors, proteins and cell patterning are possible. The disadvantage is that it is only applicable to photopolymers. ${ }^{[14-16]}$ The process isn't quick and depending on the size and number of objects being created, the laser might take a minute or two for each layer. ${ }^{[17]}$

Application: SLA models initially started to be used in medicine and dentistry for the planning of surgical procedures and as a means of constructing customized implants such as in cranioplasties, orbital floors and onlays. ${ }^{[18-25]}$ SLA is now routinely used to produce surgical guides for the placement of dental implants.

\section{Fused deposition melting (FDM)}

In this technique strands of heated polymer/ceramics are extruded through a nozzle. Fused filament fabrication is an additive manufacturing technology commonly used for modelling, prototyping, and production applications. ${ }^{[17]}$ The technology was developed by Scott Crump in the late 1980s and was commercialized in 1990. ${ }^{[27]}$ The basic principle behind this technology is that the material is passed through the heated nozzle which can move in both horizontal and vertical direction. The process is controlled digitally and the material sets immediately after extrusion from the nozzle. One of the commercially available products using this technology is 3D Bioplotter from Envisiontec. The advantage of this technique is that there is no need for a platform of support. The restriction of material due to need for molten phase stands as a disadvantage. ${ }^{[3,6,31-36]}$

Application: The main application of the bioplotter is in the modelling of scaffolds for tissue engineering and organ printing. Bioplotter from Envisiontec has the ability to use a wide range of materials, including ceramic pastes (HAP and TCP) for creating porous bone scaffolds, bioresorbable polymers such polycarpolactone and/or poly-l-lactide for drug delivery and agar, gelatin, chitosan, collagen, alginate and fibrin as carriers for cells that are used in organ printing. The bioplotter has a resolution of just a few micrometers, which means that it is able to create microstructural patterns that enhance cell invasion, proliferation, distribution and differentiation into the porous structure. ${ }^{[29]}$ Human body parts being created include blood vessels ${ }^{[30]}$, bone ${ }^{[31]}$ and soft tissue. ${ }^{[32]}$

\section{Selective Laser Sintering (SLS)}

This technique involves the use of laser to melt down the powdered material particle which then fuse together forming a 3D structure. The first step involves preparing the powder bed and then layer by layer addition of powder. Each layer is then sintered according to the CAD file using a laser source. A few materials that can be used by SLS are PCL ,Nano HA, Calcium phosphate (CaP)/poly(hydroxybutyratecohydroxyvalerate )(PHBV), Carbonated hydroxyapatite (CHAp)/poly(L-lactic acid)(PLLA), b-Tricalcium phosphate (b-TCP) PHBV. The advantages of this technique are that there is no need for support, no post processing is required. Disadvantage would be that feature resolution depends on laser beam diameter. ${ }^{[37-43]}$

\section{Additive Manufacturing: Recent Advances}

The need for reconstruction in craniofacial and skeletal bone defects due to trauma, congenital abnormalities or tumor removal is one of the main areas of application for this technology. The use of 3D scaffolds as a platform to print bone has been documented. These scaffolds are 3D-printed from fine tricalcium phosphate powder using phosphoric acid-based adhesive "ink" and soaked afterwards in aqueous sodium acetate solution for $24 \mathrm{~h}$ at $808 \mathrm{C}$ (intermediate phase - dicalcium phosphate dihydrate). ${ }^{[44]}$ The use of biomimetic materials in bone tissue engineering has opened a new venue. These materials will enable new biosurgical approaches for repairing and replacing damaged or diseased tissue, such as broken bones, damaged articular cartilage and infarcted cardiac muscle. ${ }^{[45]}$

More recently, a cranial reconstruction was performed in a patient suffering from post trauma defect after a bike accident. The defect was in the right frontal bone due to decompressive craniotomy. The defect was replaced by a printed biomodel constructed from computed tomography using Additive Manufacturing technique. Segmentaion and CAD design used to produce a digital conversion of the scanned images was done 
using the various available softwares. The biocompatible implant was fabricated using DMLS technique and was an exact fit to the patient's cranial defect. The material used was EOS Titanium Ti64 ELI (EOS, Germany). The craniotomy took 2 hours instead of three which it takes when the reconstruction plate is formulated manually. This technique proved to be successful and can be made applicable in patients suffering from large bone defects in cases of oral and maxillofacial defects. This technique can also be used in treatment planning and manufacturing of customized implants.

\section{D printing (STEREOLITHOGRAPHY) in facial surgery - use of technology and innovation for biological means}

Stereolithography, also known as 3-D layering or 3-D printing, allows you to create solid, plastic, threedimensional (3-D) objects from CAD drawings in a matter of hours. Stereolithography is a common rapid manufacturing and rapid prototyping technology for producing parts with high accuracy and good surface finish. A device that performs stereolithography is called an SLA or Stereolithography Apparatus.

\section{1) CT-guided stereolithography as a new tool in craniofacial surgery}

- Very accurate and precise impression of the structure at hand.

- treatment of certain craniofacial malformations- better visualisation of the skeletal system (direct view)

\section{Advantages}

- A reduction of the surgical risk

- measuring and touching the contours by hand

- Optimal planning of osteotomy lines and moving of bone fragments

- Exact preparation of bone grafts

- Optimal documentation of healing and growth in follow-up studies as well as for teaching purposes.

\section{2) Stereolithography in Orbital Reconstruction}

In orbital reconstruction, the relationship of bony defects to vital orbital structures must be carefully analyzed preoperatively to avoid critical surgical pitfalls and maximize the postoperative outcome. Computed tomography (CT) and magnetic resonance imaging (MRI) aid in evaluation of surgical anatomy in a multiplanar, high-resolution, two-dimensional format. Additional information may be obtained from threedimensional views.

Stereolithography modelling provides superior internal anatomic detail, surface finish, and accuracy not attainable by current milling techniques. ${ }^{[46]}$

In this way, cavities, such as sinuses, canals, and foramina, are accurately created. Using a semilucent acrylic, sinus cavities may be visualized through the SLA model ${ }^{[47]}$ This allows the surgeon to view the precise location of surgical incisions or screw placement relative to other anatomic landmarks. Multiple colours may be used in the models to delineate abnormalities in relation to normal anatomy. The surgeon may request specific dimensions of a model based on the area of the anatomy scanned.

\section{3) Head and neck oncology}

Stereolithography is helpful in orbit and head and neck oncology surgical planning and reconstruction. A tumor can be delineated on the SLA model, and the extent of the defect to be reconstructed can be visualized. The models may be used to plan osteotomy for distraction osteogenesis in bony orbital expansion surgery and for reconstructing contracted orbits after radiation therapy given during infancy. Additionally, these models allow the surgeon to bend plates preoperatively. Simulating surgical procedures preoperatively on SLA models may decrease overall treatment costs by decreasing operative time. ${ }^{[46]}$

\section{4) The potential role of stereolithography in the study of facial aging}

A number of technologies have played a role in the study of facial growth and development. Mathematical techniques for describing the 3- dimensional (3-D) morphology of the facial skeleton are becoming increasingly sophisticated. ${ }^{[48]}$ In this respect, the use of rapid prototyping offers the ability to further study craniofacial aging. Rapid prototyping, or stereolithography, was initially developed in the engineering sciences to manufacture scaled replicas of devices before assembly line production. Stereolithography uses the techniques of computer-assisted design (CAD) and computer-assisted manufacture (CAM). More recently, this technique has been applied to the fabrication of replica human skulls for treatment planning as well as for the use in anthropology. ${ }^{[9]}$

Stereolithography offers a number of potential advantages. By its nature, this technology offers a nonaveraged image with an extreme degree of accuracy. As stated, probably the single most important factor in obtaining an accurate image is a gantry position as close to $0^{\circ}$ as possible. With this position normalized, computer precision and production capabilities yield a model within $\pm 1 \mathrm{~mm}$ of the original. ${ }^{[4]]}$ 

recognized.

Perhaps the greatest potential role of stereolithography, and 3-D modelling in general, has yet to be

Some preliminary data have suggested that certain curves and contours of the facial skeleton may distort with age; stereolithography, with applied mathematics, may play an invaluable role in substantiating these observations. With significant advances being made in this field at present, stereolithography has the potential to add valuable information to the future study of facial aging.

\section{5) Stereolithography in oral and maxillofacial operation planning}

Planning reconstructive procedures in oral and maxillofacial surgery we frequently need 3-dimensional (3D) display of defects of complex cranial structures. According to the requirements in oral and maxillofacial surgery STL shows its benefits in accurate representation of complex bony structures of the entire skull for precise preparation of surgical procedures with better spatial orientation as well as in the option to manufacture implants or reconstructions prior to surgery. A further benefit is the possibility to sterilize STL models, so that they can be used during the surgical procedure.

Following are the indications for STL based operation planning in oral and maxillofacial surgery:

- Malformations, including craniofacial surgery,

- Tumor surgery,

- Traumatology,

- Orthognathic surgery,

- Implantology.

\section{6) Preoperative diagnosis of craniofacial deformities and planning of surgical corrections}

Three-dimensional models of diseases and deformities can be traced back to an old artistic tradition in medicine and surgery. The most famous examples are the "moulages" made of coloured and painted wax representing skin diseases, deformities and malignancies. However, their construction was extremely timeconsuming and only a few experts were initiated into the secrets of this profession. Until recently the only threedimensional representations of craniofacial deformities could be found in skull collections, gathered for the special purpose of illustrating and studying specific anatomic anomalies.

Three-dimensional information concerning the ideal design of the osteotomy for changing the spherical shape of the skull without creating unaesthetic steps at the skull base, and the mosaic of bone segments with optimal length, curvature and placement was "at hand" during surgery.

The simulation of transcranial hypertelorism corrections also gave essential additional information. Especially the simulation of the skull base osteotomy at the junction of the lateral aspect of the anterior and middle cerebral fossae delivered useful information concerning this delicate area of the abnormal anatomy of the shortened skull base. The simulation of the hypertelorism correction itself was also time-saving. It showed the optimal rotations of the medialized orbits taking into account aesthetic aspects such as the position of the lateral orbital rims and smooth contours of the supraorbital area and the zygomatic arches.

The most attractive aspect of the models is the perspective that the surgeon can handle a realistic model, thus enabling a three-dimensional analysis of the deformity and planning of the correction, preoperatively as well as during surgery. This is also the main reason for building a collection of stereolithographic models of rare craniomaxillofacial deformities, in order to make this unique pathology accessible for teaching and research purposes.

\section{7) Biomodelling of skull base tumours}

Neoplasms occurring in the region of the cranial base occupy the most anatomically complex and inaccessible part of the human body. Skull base anatomy leaves little room for error and at best allows only limited surgical access. Resection or debulking of such tumours is immensely challenging for the surgeon and of high risk to the patient. To minimize operative morbidity and mortality, and to maximize therapeutic success, accurate surgical strategies must be tailored to each patient and must be carefully planned using the best possible anatomical information. To optimise data display three-dimensional (3D) computed tomography (CT) technology has been used to display the skull base ${ }^{[50]}$ This technique was found to simplify interpretation, enhance communication between the radiologist and clinician, and to facilitate surgical planning. Biomodelling is the generic term that has been coined to describe the ability to replicate the morphology of a biological structure in a solid substance. Specifically, it is used to describe the process of using radiant energy to capture morphological data on a biological structure and the processing of such data by a computer to generate the code required to manufacture the structure by a rapid prototyping apparatus. A biomodel is the product of this process and real virtuality is the descriptive term coined for the visualization medium.

Several investigators have examined the use of biomodelling in cranio-maxillo-facial surgery and report clinical utility, although these studies are limited to a handful of clinical cases. ${ }^{[46]}$ 
From the limited experience biomodelling has the potential to have the following significant advantages over currently available imaging modalities for the following reasons:

- Conventional CT/MR 'slice' images are complex and require subjective reconstruction to attain 3D understanding. The accuracy of such reconstruction is dependent upon the experience and spatial aptitude of the observer. Biomodels provide a readily recognizable, tactile replica of a person's anatomy that requires no mental reconstruction.

- Biomodels optimize surgical planning and rehearsal because they may be used realistically and interactively to simulate surgery.

- Intraoperatively, biomodels may be used to simply guide the surgical approach and verify anatomical relationships without complex equipment.

- Biomodels may be used as templates for prefabrication of customized prosthetic and allographic implants improving implant design and fit, whilst reducing operative time and risk.

- Biomodels provide patients with a clearer understanding of their pathology and the aims and limitations of surgery preoperatively.

- Biomodels provide improved teaching demonstrations and allow 'hands on', interactive use of patient CT/MR data by students.

- Biomodels require no specialized equipment or knowledge for interpretation and use, are rugged and may easily be transported.

\section{Future: Application or Implications}

With the mammoth speed at with the technology is changing, a digital revolution is in order. The scope of additive manufacturing in various dental specialities especially oral and maxillofacial surgery is commendable. With increasing accuracy, speed and customization the market for 3D printing shall grow. This shall mean a better delivery of the prosthesis or implant to the patient and decreased post-operative complications. However, the availability and acceptance of this technology is far from near. Also due to lack of clinical trials and patient based research a lot of biomaterials and technology is still in the laboratories. It'll take another decade for this technology to boom. The expected surge in the market for additive manufacturing in the coming years shall prove fruitful for dentistry as well. In all, the technology of additive Manufacturing is here to stay.

\section{References}

[1]. C.W.Hull, Apparatus for production of three-dimensional objects by stereolithography, US patent\# 4, 575, 30.

[2]. F.B. PRINZ, et al. JTEC/WTEC Panel Report on Rapid Prototyping Association of the Society of Manufacturing Engineers, Loyola college in Maryland,1997.

[3]. S.Bose, S.Suguira, A. Bandhopadhyay, Scr.Matter.41 (1990)1009-1014.

[4]. S.Bose, et al.Mater.Sci. Eng. C232003) 479-486.

[5]. I. Gibson, et al, Additive Manufacturing Technologies: Rapid Prototyping to Direct Digital Manufacturing, Springer, 2009.

[6]. J. Darsell, et al. J. Am. Ceram. Soc. 87 (2003) 1076-1080.

[7]. S. Bose, et al. J. Mater. Sci.: Mater. Med. 13 (2002) 23-28.

[8]. E.M. Sachs, et al., Three-dimensional printing techniques, US Patent \#5,204,055.

[9]. E. Sachs, M.J. Cima, J. Cornie, CIRP Ann. 39/1 (1990) 204-210

[10]. P.H. Warnke, et al. J. Biomed. Mater. Res. 93B (2010) 212-217.

[11]. E. Vorndran, et al. Adv. Eng. Mater. 10 (2008) B67-B71.

[12]. B. Leukers, et al. Mater. Wiss. Werkstofftech. 36 (2005) 781-787.

[13]. R. Detsch, et al. J. Biomater. Appl. 26 (2011) 359-380.

[14]. P.X. Lan, et al. J. Mater. Sci.: Mater. Med. 20 (2009) 271-279.

[15]. J.W. Lee, et al. Microelectron. Eng. 86 (2009) 1465-1467.

[16]. A. Ronca, L. Ambrosio, D.W. Grijpma, Acta Biomater. 9 (2013) 5989-5996.

[17]. Richard van Noort, The future of dental devices is digital, Dental Materials, Volume 28, Issue 1, January 2012, Pages 312, ISSN 0109 5641,http://dx.doi.org/10.1016/j.dental.2011.10. 014.

[18]. W.G. Bian, et al. Biofabrication 3 (2011), http://dx.doi.org/10.1088/1758-5082/3/3/034103.

[19]. van Roekel NB. Electrical discharge machining in dentistry. Int J Prosthodont 1992; 5:114-21.

[20]. Winder J, Bibb R. Medical rapid prototyping technologies: state of the art and current limitations for applications in oral and maxillofacial surgery. J Oral Maxillofac Surg 2005; 63:1006-15.

[21]. Bibb R, Brown R. The application of computer aided product development techniques in medical modelling topic: rehabilitation and prostheses. Biomed Sci Instrum 2000; 36:319- 24

[22]. Joffe J, Harris M, Kahugu F, et al. A prospective study of computer-aided design and manufacture of titanium plate for cranioplasty and its clinical outcome. Br J Neurosurg 1999; 13:576-80.

[23]. Winder J, Cook RS, Gray J, et al. Medical rapid prototyping and 3D CT in the manufacture of custom made cranial titanium plates. J Med Eng Technol 1999; 23:26-8.

[24]. Bartlett P, Carter L, Russell JL. The leeds method for titanium cranioplasty construction. Brit J Oral Maxillofac Surg2009; 47:238-40.

[25]. Eufinger $\mathrm{H}$, Wehmoller $\mathrm{M}$. Individual prefabricated titanium implants in reconstructive craniofacial surgery: clinical and technical aspects of the first 22 cases. Plast Reconstruct Surg 1998; 102:300-8

[26]. http://en.wikipedia.org/wiki/Fused deposition modelling

[27]. S.J. Kalita, et al. Mater. Sci. Eng. C 23 (2003) 611-620. 
[28]. V.L. Tsang, S.N. Bhatia, Adv. Drug Deliv. Rev. 56 (2004) 1635-1647.

[29]. C.X.F. Lam, et al. Biomed. Mater. 3 (2008), http://dx.doi.org/10.1088/1748- 6041/3/3/034108.

[30]. C.X.F. Lam, et al. Polym. Int. 56 (2007) 718-728.

[31]. J.-T. Schantz, Tissue Eng. 9 (2003) S127- S139.

[32]. C.X.F. Lam, et al. J. Biomed. Mater. Res. A 90A (2009) 906-919.

[33]. Zein I, Hutmacher DW, Tan KC, Teoh SH. Fused deposition modelling of novel scaffold architectures for tissue engineering. Biomaterials 2002; 23:1169-85.

[34]. Centola M, Rainer A, Spadaccio C, et al. Combining electrospinning and fused deposition modelling for the fabrication of a hybrid vascular graft. Biofabrication 2010; 2(March (1)):014102.

[35]. Chen M, Le DQ, Baatrup A, et al. Self assembly composite matrix in a hierarchical 3D scaffold for bone tissue engineering. Acta Biomater 2011; 7(5):2244-55.

[36]. [36] Wiggenhauser PS, Muller DF, Melchels FP, et al. Engineering of vascularized adipose constructs. Cell Tissue Res 2011.August 18 [Epub ahead of print].

[37]. J.M. Williams, et al. Biomaterials 26 (2005) 4817-4827.

[38]. C. Shuai, et al. Nanotechnology 22 (2011), http://dx.doi.org/10.1088/0957-4484/22/28/285703.

[39]. B. Duan, et al. Acta Biomater. 6 (2010) 4495-4505.

[40]. S.H. Lee, et al. J. Biomimetics Biomater. Tissue Eng. 1 (2008) 81-89.

[41]. H. Qingxi, et al. International Technology and Innovation Conference, 2006.

[42]. T.F. Pereira, et al. Virtual Phys. Prototyp. 7 (2012) 275-285.

[43]. Geraldine Rodriguez, Juliana Dias, Marcos Akira d'Ávila, Paulo Bártolo, Influence of Hydroxyapatite on Extruded 3D Scaffolds, Procedia Engineering, Volume 59, 2013, Pages 263-269, ISSN 1877-7058, http://dx.doi.org/10.1016/j.proeng.2013.05.120.

[44]. Jennifer Patterson, Mikaël M. Martino, Jeffrey A. Hubbell, Biomimetic materials in tissue engineering, Materials Today, Volume 13, Issues 1-2, January-February 2010, Pages 14-22, ISSN 1369-7021, http://dx.doi.org/10.1016/S13697021(10)70013-4.

[45]. Jardini AL, Larosa MA, Maciel Filho R, Zavaglia CAdC, Bernardes LF, Lambert CS, Calderoni DR, Kharmandayan P, Cranial reconstruction: 3D biomodel and custom-built implant created using additive manufacturing, Journal of CranioMaxillo-Facial Surgery (2014), doi:10.1016/j.jcms.2014.07.006.

[46]. Yau YY, Arvier JF, Barker TM. Technical note: maxillofacial biomodelling—preliminary result. Br J Radiol 1995;68:519_ 23.

[47]. Barker TM, Earwaker WJS, Frost N, Wakeley G. Integration of 3-D medical imaging and rapid prototyping to create stereolithographic models. Australas Phys Eng Sci Med 1993;16:79-85.

[48]. Zumpano MP, Carson BS, Marsh JL, Vanderkolk CA, Richtsmeier JT. Three-dimensional morphological analysis of isolated metopic sysnostosis. Anat Rec 1999;256:177-88.

[49]. Mole C, Gerard H, Mallet JL, Chassagne JF, Miller N. A new three-dimensional treatment algorithm for complex surfaces: applications in surgery. J Oral Maxillofac Surg 1995;53:156-62.

[50]. Vannier MW, Gado MH, Marsh JL. Three-dimensional display of intracranial soft-tissue structures. Am J Neuroradiol 1983; 4: 520-521. 